\title{
Modular symmetry anomaly in magnetic flux compactification
}

\author{
Yuki Kariyazono, Tatsuo Kobayashi, Shintaro Takada, Shio Tamba, and Hikaru Uchida \\ Department of Physics, Hokkaido University, Sapporo 060-0810, Japan
}

(Received 22 April 2019; published 15 August 2019)

\begin{abstract}
We study modular symmetry anomalies in four-dimensional low-energy effective field theory, which is derived from six-dimensional supersymmetric $U(N)$ Yang-Mills theory by magnetic flux compactification. The gauge symmetry $U(N)$ is broken to $U\left(N_{a}\right) \times U\left(N_{b}\right)$ by magnetic fluxes. It is found that an Abelian subgroup of the modular symmetry corresponding to discrete part of $U(1)$ can be anomalous, but other elements independent of $U(1)$ in the modular symmetry are always anomalyfree.
\end{abstract}

DOI: 10.1103/PhysRevD.100.045014

\section{INTRODUCTION}

The modular symmetry is a geometrical feature, which torus compactification as well as orbifold compactification has. Furthermore, the modular symmetry plays an important role in four-dimensional (4D) low-energy effective field theory derived from higher dimensional field theory and superstring theory.

The modular symmetry in string-derived supergravity theory was studied in Ref. [1] and also its anomaly was studied in Ref. [2,3]. (See also for anomalies in explicit heterotic orbifold models Ref. [4].) Recently, these studies were extended to supergravity theory derived by magnetized and intersecting D-brane models [5]. Furthermore, their anomalies are also interesting from the phenomenological viewpoint $[3,6,7]$.

Also it was studied how massless modes transform under modular symmetry in heterotic orbifold models [8-10]. Recently, modular transformation behavior of massless modes was studied in magnetized D-brane models as well as intersecting D-brane models [11-14]. Then, it was found that the modular symmetry transforms massless modes each other, and that is a sort of flavor symmetries. On the other hand, it was shown that nonAbelian discrete flavor symmetries appear in heterotic orbifold models [15-20] and magnetized/intersecting D-brane models [21-26] through analysis independent of modular symmetry. Indeed, a relation between modular symmetry and non-Abelian discrete flavor symmetry was also studied [13].(See also Ref. [27].)

Non-Abelian discrete flavor symmetries are interesting from the phenomenological viewpoints [28-30]. Various

Published by the American Physical Society under the terms of the Creative Commons Attribution 4.0 International license. Further distribution of this work must maintain attribution to the author(s) and the published article's title, journal citation, and DOI. Funded by SCOAP ${ }^{3}$. finite groups have been utilized such as $S_{3}, A_{4}, S_{4}, A_{5}$, etc. for $4 \mathrm{D}$ field-theoretical model building. Then, many models have been proposed in order to realize quark and lepton masses and their mixing angles and $C P$ phases. The modular group includes $S_{3}, A_{4}, S_{4}, A_{5}$ as its finite subgroups [31]. This aspect in addition to the above string compactification inspired a new approach of 4D fieldtheoretical model building [32], where finite subgroups of the modular symmetry are used as non-Abelian discrete flavor symmetries and also couplings and masses are assumed to transform nontrivially under such finite subgroups. Such a new approach has been applied to models with $S_{3}, A_{4}, S_{4}, A_{5}$ modular symmetries [33-41].

Thus, the modular symmetry is important from both theoretical and phenomenological viewpoints. In general, continuous and discrete symmetries can be anomalous. (See for anomalies of Abelian and non-Abelian discrete symmetries Refs. [42-45].) Anomalous symmetries can be broken by nonperturbative effects. That is, breaking terms are induced by nonperturbative effects. Such breaking terms may have important implications. The purpose of this paper is to study the anomaly structure of the modular symmetry in 4D low-energy effective field theory derived from magnetic flux compactification of higher dimensional supersymmetric Yang-Mills theory, which is effective field theory of magnetized D-brane models.

This paper is organized as follows. In Sec. II, we present our setup and give a brief review on magnetic flux compactification and the modular transformation of zeromodes. In Sec. III, we study the anomaly structure of the modular symmetry. Section IV is our conclusion.

\section{MODULAR TRANSFORMATION OF MAGNETIC FLUX COMPACTIFICATION}

\section{A. Setup and wave functions}

Here, we present our setup and give a brief review on magnetic flux compactification. We start with six-dimensional 
supersymmetric $U(N)$ Yang-Mills theory, which can be derived from D-brane system. Then, we consider the twodimensional torus $T^{2}$ compactification with magnetic flux. Similarly, we can study higher dimensional theory such as ten-dimensional supersymmetric Yang-Mills theory on $T^{2} \times T^{2} \times T^{2}$, which can also be derived from D-brane system. Indeed, magnetic flux compactification on $T^{2}$ leads to tachyonic modes. On the other hand, tachyonic modes can be avoided on $T^{2} \times T^{2} \times T^{2}$ by choosing proper combinations of magnetic fluxes and sizes of three $T^{2}$ tori. However, each $T^{2}$ tours is important to study the anomaly structure. Thus, here we concentrate on the $T^{2}$ compactification by assuming absence of tachyonic modes in $T^{2} \times T^{2} \times T^{2}$.

We use the complex coordinate $z=x^{1}+\tau x^{2}$ on $T^{2}$, where $\tau$ is the complex modulus parameter, and $x^{1}$ and $x^{2}$ are real coordinates. The metric on $T^{2}$ is given by

$$
g_{\alpha \beta}=\left(\begin{array}{ll}
g_{z z} & g_{z \bar{z}} \\
g_{\bar{z} z} & g_{\bar{z} \bar{z}}
\end{array}\right)=(2 \pi R)^{2}\left(\begin{array}{cc}
0 & \frac{1}{2} \\
\frac{1}{2} & 0
\end{array}\right) \text {. }
$$

We identify $z \sim z+1$ and $z \sim z+\tau$ on $T^{2}$.

We introduce the following magnetic flux along the diagonal direction,

$$
F=i \frac{\pi}{\operatorname{Im} \tau}(d z \wedge d \bar{z})\left(\begin{array}{cc}
M_{a} \mathbb{I}_{N_{a} \times N_{a}} & 0 \\
0 & M_{b} \mathbb{I}_{N_{b} \times N_{b}}
\end{array}\right),
$$

where $N_{a}+N_{b}=N, \mathbb{I}_{N_{a, b} \times N_{a, b}}$ denotes the $\left(N_{a, b} \times N_{a, b}\right)$ identity matrix and $M_{a, b}$ must be integer. This form of magnetic flux corresponds to the vector potential,

$$
A(z)=\frac{\pi}{\operatorname{Im} \tau} \operatorname{Im}(\bar{z} d z)\left(\begin{array}{cc}
M_{a} \mathbb{I}_{N_{a} \times N_{a}} & 0 \\
0 & M_{b} \mathbb{I}_{N_{b} \times N_{b}}
\end{array}\right) .
$$

Because of this gauge background, the $U(N)$ gauge symmetry breaks to $U\left(N_{a}\right) \times U\left(N_{b}\right)$.

Now let us study the gaugino sector. The spinor field on $T^{2}$ has two components, $\lambda_{ \pm}$. They are decomposed to

$$
\left(\begin{array}{cc}
\lambda_{ \pm}^{a a} & \lambda_{ \pm}^{a b} \\
\lambda_{ \pm}^{b a} & \lambda_{ \pm}^{b b}
\end{array}\right)
$$

Here $\lambda^{a a}$ and $\lambda^{b b}$ correspond to the gaugino fields of unbroken gauge groups, $U\left(N_{a}\right)$ and $U\left(N_{b}\right)$, respectively, while $\lambda^{a b}$ and $\lambda^{b a}$ correspond to $\left(N_{a}, \bar{N}_{b}\right)$ and $\left(\bar{N}_{a}, N_{b}\right)$ under $U\left(N_{a}\right) \times U\left(N_{b}\right)$.

The zero-mode equation with the above gauge background (3),

$$
i \not D \lambda_{ \pm}=0
$$

has chiral solutions. When $M=M_{a}-M_{b}$ is positive, $\lambda_{+}^{a b}$ and $\lambda_{-}^{b a}$ have $M$ degenerate zero-modes, ${ }^{1}$ whose profiles are written by [11]

$$
\psi_{T^{2}}^{j, M}(z)=\mathcal{N} e^{i \pi M z_{\frac{\mathrm{Im}}{\mathrm{m} \tau} \tau}} \cdot \vartheta\left[\begin{array}{c}
\frac{j}{M} \\
0
\end{array}\right](M z, M \tau),
$$

with $j=0,1, \ldots,(M-1)$, where $\vartheta$ denotes the Jacobi theta function,

$$
\vartheta\left[\begin{array}{l}
a \\
b
\end{array}\right](\nu, \tau)=\sum_{l \in \mathbf{Z}} e^{\pi i(a+l)^{2} \tau} e^{2 \pi i(a+l)(\nu+b)} .
$$

Here, $\mathcal{N}$ denotes the normalization factor given by

$$
\mathcal{N}=\left(\frac{2 \operatorname{Im} \tau M}{\mathcal{A}^{2}}\right)^{1 / 4}
$$

with $\mathcal{A}=4 \pi^{2} R^{2} \operatorname{Im} \tau$.

On the other hand, when $M$ is negative, $\lambda_{-}^{a b}$ and $\lambda_{+}^{b a}$ have $|M|$ degenerate zero-modes, whose profiles are the same as $\psi^{j, M}(z)$ except $M$ replacing by $|M|$. Hereafter, we set $M$ to be positive. That is, we consider the model that has $M$ degenerate zero-modes of $\lambda_{+}^{a b}$ and $\lambda_{-}^{b a}$, but no zero-modes of $\lambda_{-}^{a b}$ and $\lambda_{+}^{b a}$.

Because of the chiral spectrum, $U(1)_{a}$ and $U(1)_{b}$ are anomalous in $4 \mathrm{D}$ low-energy effective field theory. For example, both the mixed anomalies, $U(1)_{a}-S U\left(N_{b}\right)^{2}$ and $U(1)_{b}-S U\left(N_{a}\right)^{2}$ are proportional to $M$. Such anomalies can be canceled by the Green-Schwarz mechanism, if we include the Green-Schwarz field in our theory. The GreenSchwarz mechanism cancels anomalies by the shift of axions $\chi_{a, b}$,

$$
\chi_{a, b} \rightarrow \chi_{a, b}+\alpha_{a, b},
$$

under $U(1)_{a, b}$ transformation, where $\alpha_{a, b}$ are $U(1)_{a, b}$ gauge transformation parameters [46]. Those axions are eaten by $U(1)_{a, b}$ gauge bosons and then $U(1)_{a, b}$ gauge bosons become massive.

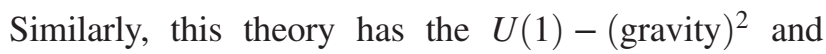
$U(1)^{3}$ anomalies. Those can also be canceled by the Green Schwarz mechanism.

In the next section, we will study the $T^{2} / Z_{2}$ orbifold background. For simplicity, we focus on orbifold models without Wilson lines. The zero-mode wave functions on $T^{2} / Z_{2}$ are obtained from the above wave functions [47]. The above wave functions have the following property:

\footnotetext{
${ }^{1}$ Note that the six-dimensional chirality is fixed. Then, $\lambda_{+}^{a b}$ and $\lambda_{-}^{b a}$ are combined with 4D left-handed and right-handed spinor fields, and they correspond to a pair of matter and antimatter.
} 


$$
\psi_{T^{2}}^{j, M}(-z)=\psi_{T^{2}}^{M-j, M}(z) .
$$

Thus, the $T^{2}$ wave function with $j=0$ is still the $Z_{2}$-even zero-mode on $T^{2} / Z_{2}$. Also, when $M=$ even, the $T^{2}$ wave function with $j=M / 2$ is still the $Z_{2}$-even zero-mode on $T^{2} / Z_{2}$. That is, we obtain

$$
\psi_{T^{2} / Z_{2}^{+}}^{j, M}(z)=\psi_{T^{2}}^{j, M}(z)
$$

for $j=0, M / 2$. For the other, the $Z_{2}$-even and odd zeromodes can be written by

$$
\psi_{T^{2} / Z_{2}^{ \pm}}^{j, M}(z)=\frac{1}{\sqrt{2}}\left(\psi_{T^{2}}^{j, M}(z) \pm \psi_{T^{2}}^{M-j, M}(z)\right) .
$$

When $M=$ even, totally the numbers of $Z_{2}$-even and odd zero-modes are equal to $(M / 2+1)$ and $(M / 2-1)$, respectively. When $M=$ odd, the numbers of $Z_{2}$-even and odd zero-modes are equal to $((M-1) / 2+1)$ and $((M-1) / 2)$, respectively. Either $Z_{2}$ even or odd modes are projected out by the $Z_{2}$ projection.

The anomalies of $U(1)_{a}$ and $U(1)_{b}$ on the $T^{2} / Z_{2}$ orbifold, e.g., for the $Z_{2}$-even modes $\psi_{T^{2} / Z_{2}^{+}}^{j, M}(z)$, can be studied in the same way as on the torus. Those anomalies can also be canceled by the Green-Schwarz mechanism.

\section{B. Modular transformation}

Here, we give a brief review on modular transformation of zero-mode wave functions [11-14]. Following [12], we restrict ourselves to even magnetic fluxes $M$.

Under the modular transformation, the modulus $\tau$ transforms as

$$
\tau \rightarrow \frac{a \tau+b}{c \tau+d} .
$$

This group includes two important generators, $S$ and $T$,

$$
\begin{gathered}
S: \tau \rightarrow-\frac{1}{\tau}, \\
T: \tau \rightarrow \tau+1 .
\end{gathered}
$$

The generator $S$ transforms the zero-mode wave functions as

$$
\psi^{j, M} \rightarrow \frac{1}{\sqrt{M}} \sum_{k} e^{2 \pi i j k / M} \psi^{k, M} .
$$

On the other hand, the generator $T$ transforms the zeromode wave functions

$$
\psi^{j, M} \rightarrow e^{\pi i j^{2} / M} \psi^{j, M} .
$$

Generically, the $T$ generator satisfies [12]

$$
T^{2 M}=\mathbb{I}_{M \times M},
$$

on the zero-modes, $\psi^{j, M}$. Furthermore, in Ref. [12] it is shown that

$$
(S T)^{3}=e^{\pi i / 4} \mathbb{I}_{M \times M},
$$

on the zero-modes, $\psi^{j, M}$. Hence, $T$ and $(S T)^{3}$ are represented by diagonal matrices on $\psi^{j, M}$, and they are $Z_{2 M}$ and $Z_{8}$ symmetries, respectively.

The above representations of $S$ and $T$ on $\psi^{j, M}$ are reducible. It is obvious that $\psi^{j, M}$ and $\psi^{M-j, M}$ transform in the same way under both $S$ and $T$. That implies that the orbifold basis $\psi_{T^{2} / Z_{2}^{ \pm}}^{j, M}(z)$ corresponds to the irreducible representation. We denote such irreducible representations by $S_{ \pm}$and $T_{ \pm}$. Their explicit forms can be read off from the above representations of $S$ and $T$. Note that when $M=$ even, $S_{+}$and $T_{+}$are $(M / 2+1) \times(M / 2+1)$ matrices, and $S_{-}$and $T_{-}$are $(M / 2-1) \times(M / 2-1)$ matrices.

\section{MODULAR SYMMETRY ANOMALY}

Here, we study the modular symmetry anomaly. Anomalies of non-Abelian discrete symmetries were studied in Ref. [45]. Each element of a non-Abelian discrete group, $g$, generates Abelian discrete symmetry, $Z_{K}$ i.e., $g^{K}=1$. Thus, basically anomalies of non-Abelian discrete group are studied by analyzing Abelian discrete anomalies of each element, $g$. However, states correspond to a multiplet under a non-Abelian discrete symmetry. That is, $g$ is represented by a matrix. Suppose that zero-modes correspond to the (anti-)fundamental representation of $S U\left(N_{b}\right)$. Then, if $\operatorname{det} g=1$, the mixed $Z_{K}-S U\left(N_{b}\right)^{2}$ anomaly vanishes. Otherwise, the $Z_{K}$ symmetry generated by $g$ can be anomalous. Furthermore, suppose that zero-modes correspond to the bifundamental representation $\left(N_{a}, \bar{N}_{b}\right)$ under $S U\left(N_{a}\right) \times S U\left(N_{b}\right)$. Then, if $\operatorname{det} g^{N_{a}}=1$, the mixed $Z_{K}-S U\left(N_{b}\right)^{2}$ anomaly vanishes. Otherwise, the $Z_{K}$ symmetry generated by $g$ is anomalous. Hence, the quantity $\operatorname{det} g$ is important to examine anomalies. If $\operatorname{det} g \neq 1$, such discrete symmetry can be anomalous. Also, we can study $Z_{K}-(\text { gravity })^{2}$ anomalies. If $\operatorname{det} g=1$, such elements do not contribute to gravitational mixed anomalies.

\section{A. $T^{2} / Z_{2}$ orbifold}

As mentioned above, the orbifold basis is more fundamental. Thus, we first study anomalies due to the $Z_{2}$-even modes on the $T^{2} / Z_{2}$ orbifold. Here, we study anomalies by examining $\operatorname{det} g$ for smaller $M$ concretely.

$$
\text { 1. } M=2
$$

Here, we study the modular symmetry for $M=2$. Note that the zero-modes on $T^{2}$ are the same as the $Z_{2}$-even zeromodes on $T^{2} / Z_{2}$. First, we study diagonal elements, $T$ and $(S T)^{3}$. Their explicit forms are written as 


$$
T_{(2)}=\left(\begin{array}{cc}
1 & \\
& i
\end{array}\right), \quad\left(S_{(2)} T_{(2)}\right)^{3}=e^{\pi i / 4} \mathbb{I}_{2 \times 2},
$$

where we have omitted vanishing off-diagonal entries. That is the $Z_{4} \times Z_{8}$ symmetry, and they satisfy $\operatorname{det} T_{(2)} \neq 1$ and $\operatorname{det}\left(S_{(2)} T_{(2)}\right)^{3} \neq 1$. Thus, both symmetries can be anomalous. However, their combination,

$$
T_{(2)}^{\prime}=T_{(2)}\left(S_{(2)} T_{(2)}\right)^{-3}=\left(\begin{array}{ll}
e^{-\pi i / 4} & \\
& e^{\pi i / 4}
\end{array}\right),
$$

has $\operatorname{det} T_{(2)}^{\prime}=1$ and is always anomalyfree. This is the $Z_{8}$ symmetry. Hence, the $Z_{4} \times Z_{8}$ symmetry can be broken to $Z_{8}$ by anomalies. The generator $A_{(2)}=\left(S_{(2)} T_{(2)}\right)^{3}$ can be anomalous. Note that $\left(A_{(2)}\right)^{4}=\left(T_{(2)}^{\prime}\right)^{4}$. It is obvious that $A_{(2)}$ is commutable with any element. Therefore, at least the elements $\left(A_{(2)}\right)^{k} g(k=1,2,3)$ with $\operatorname{det} g=1$ has $\operatorname{det}\left(\left(A_{(2)}\right)^{k} g\right) \neq 1$ and can be anomalous among all of the elements, which are generated by $S_{(2)}$ and $T_{(2)}$. Indeed, explicit calculation shows that the order of the full group generated by $S_{(2)}$ and $T_{(2)}$ is equal to 192, and among them the number of elements with $\operatorname{det} g=1$ is equal to 48 . Thus, all of the elements with $\operatorname{det} h \neq 1$ can be written by $h=\left(A_{(2)}\right)^{k} g(k=1,2,3)$ with $\operatorname{det} g=1$. That is, only the element $A_{(2)}$ is important for anomalies.

The element $A_{(2)}$ can be anomalous. For example, it can lead to the mixing anomalies with $S U\left(N_{a}\right)$ and $S U\left(N_{b}\right)$. However, it is remarkable that the element $A_{(2)}$ corresponds to a subgroup of $U(1)_{a}$ as well as $U(1)_{b}$. Thus, when we include the Green-Schwarz field in our theory in order to cancel $U(1)$ anomalies, the discrete anomalies corresponding to $A_{(2)}$ can also be canceled by the same Green-Schwarz mechanism as one for $U(1)_{a}$ and $U(1)_{b}$. The other discrete parts, which are independent of $A_{(2)}$, are always anomalyfree.

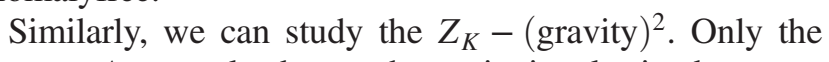
element $A_{(2)}$ can lead to such gravitational mixed anomalies, because the others have $\operatorname{det} g=1$. Such anomalies can be canceled by the same Green-Schwarz mechanism as one for $U(1)_{a}$ and $U(1)_{b}$.

The $\left(Z_{K}\right)^{3}$ anomaly has a clear meaning only if $Z_{K}$ originates from $U(1)$ group [42-45]. ${ }^{2}$ Thus, the relation between $\left(Z_{K}\right)^{3}$ and $U(1)^{3}$ anomalies as well as their GreenSchwarz cancellation mechanisms is rather clear when $Z_{K}$

\footnotetext{
${ }^{2}$ When we examine anomalies by the Feynman diagram calculations, we use currents associated with symmetries, but we can not define currents for discrete symmetries. On the other hand, we can examine anomalies of discrete symmetries by the path integral approach. Then, anomalies appear as mixed anomalies between a discrete symmetry and gauge symmetries (gravity), whose gauge bosons (gravitons) are included in covariant derivatives of fermions.
}

is the subgroup of $U(1)$. For the other part, we do not discuss the $\left(Z_{K}\right)^{3}$ anomaly.

As mentioned in the previous section, in the GreenSchwarz mechanism the axion $\chi$ shifts under the $U(1)$ transformation to cancel anomalies. Such an axion is the pure imaginary part of a complex field $U$ in the supersymmetric theory, where axionic shift (9) leads to $U \rightarrow$ $U+i \alpha$ under the $U(1)$ gauge transformation with the transformation parameter $\alpha$. It implies that $e^{-c U}$ transforms linearly and it behaves as if it has the $U(1)$ "charge" $-c$. Nonperturbative effects such as D-brane instanton effects induce new terms $e^{-c U} \phi_{1} \phi_{2} \cdots$ in 4D low-energy effective field theory. Such terms are invariant under the anomalous $U(1)$ and discrete symmetry with taking into account the transformation of $e^{-c U}$. However, when we replace $U$ by its vacuum expectation value, such terms correspond to breaking terms. Thus, breaking terms for anomalous symmetries appear. Similar breaking terms would also appear by fieldtheoretical instanton effects even if we do not take string nonperturbative effects into account.

\section{2. $M=4$}

Similarly, we study the orbifold model with $M=4$, in particular the $Z_{2}$-even modes. First, we study diagonal elements, $T$ and $(S T)^{3}$. Their explicit forms are written as

$T_{(4)+}=\left(\begin{array}{ccc}1 & & \\ & e^{\pi i / 4} & \\ & & -1\end{array}\right), \quad\left(S_{(4)+} T_{(4)+}\right)^{3}=e^{\pi i / 4} \mathbb{I}_{3 \times 3}$.

They correspond to the $Z_{8} \times Z_{8}$ symmetry. We find that $\operatorname{det} T_{(4)+} \neq 1$ and $\operatorname{det}\left(S_{(4)+} T_{(4)+}\right)^{3} \neq 1$. They can be anomalous. However, their combination,

$T_{(4)+}^{\prime}=T_{(4)+}\left(S_{(4)+} T_{(4)+}\right)^{3}=\left(\begin{array}{lll}e^{\pi i / 4} & & \\ & e^{2 \pi i / 4} & \\ & & e^{5 \pi i / 4}\end{array}\right)$,

has $\operatorname{det} T_{(4)+}^{\prime}=1$, and is always anomalyfree. This is the $Z_{8}$ symmetry. The $Z_{8} \times Z_{8}$ symmetry can be broken to $Z_{8}$ by anomalies. The generator $A_{(4)}=\left(S_{(4)+} T_{(4)+}\right)^{3}=e^{\pi i / 4} \mathbb{I}_{3 \times 3}$ can be anomalous again, and this is commutable with any element. At least the elements $\left(A_{(4)+}\right)^{k} g(k=1, \ldots, 7)$ with $\operatorname{det} g=1$ has $\operatorname{det}\left(\left(A_{(4)+}\right)^{k} g\right) \neq 1$ and can be anomalous among all of the elements, which are generated by $S_{(4)+}$ and $T_{(4)+}$. Indeed, explicit calculation shows that the order of the full group generated by $S_{(4)+}$ and $T_{(4)+}$ is equal to 768 , and among them the number of elements with $\operatorname{det} g=1$ is equal to 96 . Thus, all of the elements with det $h \neq 1$ can be written by $h=\left(A_{(4)+}\right)^{k} g(k=1, \ldots, 7)$ with $\operatorname{det} g=1$. 
The generator $A_{(4)+}$ is a subelement of $U(1)_{a}$ as well as $U(1)_{b}$. Thus, anomalies originated from $A_{(4)+}$ can be canceled by the Green-Schwarz mechanism.

\section{3. $M=6$}

Similarly, we study the orbifold model with $M=6$, in particular the $Z_{2}$-even modes. The diagonal elements, $T$ and $(S T)^{3}$, are explicitly written by

$$
\begin{aligned}
T_{(6)+} & =\left(\begin{array}{cccc}
1 & & & \\
& e^{\pi i / 6} & & \\
& & e^{2 \pi i / 3} & \\
& & e^{3 \pi i / 2}
\end{array}\right), \\
\left(S_{(6)+} T_{(6)+}\right)^{3} & =e^{\pi i / 4} \mathbb{I}_{4 \times 4},
\end{aligned}
$$

where $\operatorname{det}\left(S_{(6)+} T_{(6)+}\right)^{3}=-1$. They correspond to the $Z_{12} \times Z_{8}$ symmetry. They can be anomalous. By their combinations, we can construct the diagonal elements with $\operatorname{det} g=1$ such as

$$
\begin{aligned}
\left(S_{(6)+} T_{(6)+}\right)^{6} & =i \mathbb{I}_{4 \times 4}, \\
\left(T_{(6)+}\right)^{3}\left(S_{(6)+} T_{(6)+}\right)^{3} & =\left(\begin{array}{llll}
e^{\pi i / 4} & & & \\
& e^{3 \pi i / 4} & & \\
& & e^{\pi i / 4} & \\
& & & e^{3 \pi i / 4}
\end{array}\right),
\end{aligned}
$$

etc. They include $T_{(6)+}^{k}$ only for $k=3 k^{\prime}$ with $k^{\prime}=$ integer, but the elements $g$ including $T_{(6)+}^{k}$ for $k=3 k^{\prime}+1$ and $k=$ $3 k^{\prime}+2$ have det $g \neq 1$ and can be anomalous. The order of the above group with det $g=1$ in the $Z_{12} \times Z_{8}$ symmetry is equal to 16 . Thus, its order reduces by the factor $1 / 6$. Indeed, the order of the full group generated by $S_{(6)+}$ and $T_{(6)+}$ is equal to 2304 , and among them the number of elements with $\operatorname{det} g=1$ is equal to 384 . That is, the order reduces by the factor $1 / 6$. Here, it seems that the group elements including $T_{(6)+}^{k}$ with $k=1,2$ in addition $\left(S_{(6)+} T_{(6)+}\right)^{3}$ can be anomalous. That is different from the above cases with $M=2$ and 4.

However, $\left(S_{(6)+} T_{(6)+}\right)^{3}$ corresponds to the subelement of $U(1)_{a, b}$. Let us combine $T_{(6)+}$ and a discrete transformation of $U(1)_{a, b}$,

$$
T_{(6)+}^{\prime}=e^{i \alpha} T_{(6)+} \cdot
$$

When $\alpha=-1 / 12$, we have $\operatorname{det} T_{(6)+}^{\prime}=1$, and $T_{(6)+}^{\prime}$ is written explicitly as

$$
T_{(6)+}^{\prime}=\left(\begin{array}{llll}
e^{-\pi i / 12} & & & \\
& e^{\pi i / 12} & & \\
& & e^{7 \pi i / 12} & \\
& & & e^{-7 \pi i / 12}
\end{array}\right)
$$

As a result, in the comprehensive symmetry including the modular symmetry and $U(1)_{a, b}$, only $U(1)_{a, b}$ including their discrete symmetries can be anomalous. In this sense, the anomaly structure for $M=6$ is the same as the previous examples for $M=2$ and $M=4$, where only discrete symmetries of $U(1)_{a, b}$ as well as of course $U(1)_{a, b}$ themselves can be anomalous.

\section{Larger $M$}

Similarly, we can study anomalies for larger $M$. The anomaly structure for larger $M$ is the same as one for $M=2,4,6$. For $M \neq 6 k, T_{(M)+}$ and $\left(S_{(M)+} T_{(M)+}\right)^{3}$, in general, have $\operatorname{det} T_{(M)+} \neq 1$ and $\operatorname{det}\left(S_{(M)+} T_{(M)+}\right)^{3} \neq 1$, although in specific values of $M$ we have $\operatorname{det} T_{(M)+}=1$ for $(M+1)(M / 2+1)=24 k^{3}$ and $\operatorname{det}\left(S_{(M)+} T_{(M)+}\right)^{3}=1$ for $M=16 k-2$. However, we can find the element $T_{(M)+}^{\prime}=T_{(M)+}\left(S_{(M)+} T_{(M)+}\right)^{3 m}$ satisfying $\operatorname{det} T_{(M)+}^{\prime}=1$. Then, only the element $\left(S_{(M)+} T_{(M)+}\right)^{3}$ can be anomalous. That is, only the discrete symmetry of $U(1)_{a, b}$ can be anomalous.

For $M=6 k$, even if we combine $T_{(M)+}^{\ell}$ and $\left(S_{(M)+} T_{(M)+}\right)^{3 m}$, there are elements with $\operatorname{det} g \neq 1$ except $\left(S_{(M)+} T_{(M)+}\right)^{3 m}$. However, we can obtain $T_{(M)+}^{\prime}=$ $e^{i \alpha} T_{(M)+}$ with $\operatorname{det} T_{(M)+}^{\prime}=1$ by combining $T_{(M)+}$ with a proper discrete element of $U(1)_{a, b}$.

As a result, it is found that only the $U(1)_{a, b}$ including their discrete symmetries can be anomalous, but the other symmetries independent of $U(1)_{a, b}$ are always anomalyfree.

Although we have studied anomalies for the $Z_{2}$ even modes, we can study similarly anomalies for the $Z_{2}$ odd modes. One example is shown in the next subsection. Note that either $Z_{2}$ even or odd modes are projected out in $T^{2} / Z_{2}$ orbifold models, but both appear in $T^{2}$ models.

\section{B. $T^{2}$}

Similarly, we can discuss $T^{2}$ models. The zero-modes of $T^{2}$ are combinations of $Z_{2}$-even and odd modes on the $T^{2} / Z_{2}$ orbifold. For $M=2$, all of the zero-modes on $T^{2}$ are the $Z_{2}$-even zero-modes. Thus, $S$ and $T$ are represented by $S_{(2)}$ and $T_{(2)}$.

For $M=4$, there is one $Z_{2}$-odd mode. Then, the diagonal elements, $T$ and $(S T)^{3}$ are represented by

\footnotetext{
${ }^{3} M$ is obtained by $M=16 n-2$ with $n$ satisfying $n(16 n-1)=3 k$.
} 


$$
\begin{aligned}
T_{(4)} & =\left(\begin{array}{ll}
T_{(4)+} & \\
& T_{(4)-}
\end{array}\right), \\
\left(S_{(4)} T_{(4)}\right)^{3} & =\left(\begin{array}{ll}
\left(S_{(4)+} T_{(4)+}\right)^{3} & \\
& \left(S_{(4)-} T_{(4)-}\right)^{3}
\end{array}\right),
\end{aligned}
$$

where $T_{(4)-}=e^{\pi i / 4}$ and $\left(S_{(4)-} T_{(4)-}\right)^{3}=e^{\pi i / 4}$. That is, we have $\left(S_{(4)} T_{(4)}\right)^{3}=e^{\pi i / 4} \mathbb{I}_{4 \times 4}$. This element corresponds to the discrete subgroup of $U(1)_{a, b}$ and can be anomalous. Other elements independent of $U(1)_{a, b}$ discrete subgroup are always anomalyfree. For example, from $T_{(4)}$ we can construct $T_{(4)}^{\prime}=e^{i \alpha} T_{(4)}$ with $\operatorname{det} T_{(4)}^{\prime}=1$ by choosing a proper value of $\alpha$.

\section{CONCLUSION}

We have studied the modular symmetry anomalies in magnetic flux compactifiction. Our model is six-dimensional supersymmetric $U(N)$ Yang-Mills theory, where
$U(N)$ gauge symmetry is broken down to $U\left(N_{a}\right) \times$ $U\left(N_{b}\right)$ by magnetic fluxes in the compact space. Discrete subsymmetries of $U(1)_{a, b}$ in the modular symmetry can be anomalous, but other discrete elements, which are independent of $U(1)_{a, b}$, are always anomalyfree. Anomalies of such discrete symmetries can be canceled by the same Green-Schwarz mechanism as the mechanism to cancel $U(1)_{a, b}$ anomalies. As a result, breaking terms can be induced only for continuous and discrete $U(1)_{a, b}$ symmetries.

Here we have studied supersymmetric $U(N)$ Yang-Mills theory, which can be derived from D-brane models. Similar representations of $S$ and $T$ were derived in heterotic orbifold models [8-10]. It is interesting to carry out a similar analysis on heterotic orbifold models.

\section{ACKNOWLEDGMENTS}

T. K. was supported in part by MEXT KAKENHI Grant No. JP19H04605.
[1] S. Ferrara, D. Lust, A. D. Shapere, and S. Theisen, Phys. Lett. B 225, 363 (1989).

[2] J. P. Derendinger, S. Ferrara, C. Kounnas, and F. Zwirner, Nucl. Phys. B372, 145 (1992).

[3] L. E. Ibanez and D. Lust, Nucl. Phys. B382, 305 (1992).

[4] T. Araki, K. S. Choi, T. Kobayashi, J. Kubo, and H. Ohki, Phys. Rev. D 76, 066006 (2007).

[5] T. Kobayashi, S. Nagamoto, and S. Uemura, Prog. Theor. Exp. Phys. 2017, 023B02 (2017).

[6] L. E. Ibanez, D. Lust, and G. G. Ross, Phys. Lett. B 272, 251 (1991).

[7] H. Kawabe, T. Kobayashi, and N. Ohtsubo, Nucl. Phys. B434, 210 (1995).

[8] J. Lauer, J. Mas, and H. P. Nilles, Phys. Lett. B 226, 251 (1989); Nucl. Phys. B351, 353 (1991).

[9] W. Lerche, D. Lust, and N. P. Warner, Phys. Lett. B 231, 417 (1989).

[10] S. Ferrara, D. Lust, and S. Theisen, Phys. Lett. B 233, 147 (1989).

[11] D. Cremades, L. E. Ibanez, and F. Marchesano, J. High Energy Phys. 05 (2004) 079.

[12] T. Kobayashi and S. Nagamoto, Phys. Rev. D 96, 096011 (2017).

[13] T. Kobayashi, S. Nagamoto, S. Takada, S. Tamba, and T. H. Tatsuishi, Phys. Rev. D 97, 116002 (2018).

[14] T. Kobayashi and S. Tamba, Phys. Rev. D 99, 046001 (2019).

[15] T. Kobayashi, H. P. Nilles, F. Ploger, S. Raby, and M. Ratz, Nucl. Phys. B768, 135 (2007).

[16] T. Kobayashi, S. Raby, and R. J. Zhang, Nucl. Phys. B704, 3 (2005).
[17] P. Ko, T. Kobayashi, J. h. Park, and S. Raby, Phys. Rev. D 76, 035005 (2007); 76, 059901(E) (2007).

[18] F. Beye, T. Kobayashi, and S. Kuwakino, Phys. Lett. B 736, 433 (2014).

[19] Y. Olguin-Trejo, R. Perez-Martinez, and S. Ramos-Sanchez, Phys. Rev. D 98, 106020 (2018).

[20] H. P. Nilles, M. Ratz, A. Trautner, and P. K. S. Vaudrevange, Phys. Lett. B 786, 283 (2018).

[21] H. Abe, K. S. Choi, T. Kobayashi, and H. Ohki, Nucl. Phys. B820, 317 (2009).

[22] H. Abe, K. S. Choi, T. Kobayashi, and H. Ohki, Phys. Rev. D 80, 126006 (2009); 81, 126003(E) (2010).

[23] M. Berasaluce-Gonzalez, P. G. Camara, F. Marchesano, D. Regalado, and A. M. Uranga, J. High Energy Phys. 09 (2012) 059.

[24] F. Marchesano, D. Regalado, and L. Vazquez-Mercado, J. High Energy Phys. 09 (2013) 028.

[25] H. Abe, T. Kobayashi, H. Ohki, K. Sumita, and Y. Tatsuta, J. High Energy Phys. 06 (2014) 017.

[26] T. Higaki, N. Kitazawa, T. Kobayashi, and K. j. Takahashi, Phys. Rev. D 72, 086003 (2005).

[27] A. Baur, H. P. Nilles, A. Trautner, and P. K. S. Vaudrevange, Phys. Lett. B 795, 7 (2019).

[28] G. Altarelli and F. Feruglio, Rev. Mod. Phys. 82, 2701 (2010).

[29] H. Ishimori, T. Kobayashi, H. Ohki, Y. Shimizu, H. Okada, and M. Tanimoto, Prog. Theor. Phys. Suppl. 183, 1 (2010); Lect. Notes Phys. 858, 1 (2012).

[30] S. F. King and C. Luhn, Rep. Prog. Phys. 76, 056201 (2013). 
[31] R. de Adelhart Toorop, F. Feruglio, and C. Hagedorn, Nucl. Phys. B858, 437 (2012).

[32] F. Feruglio, arXiv:1706.08749.

[33] T. Kobayashi, K. Tanaka, and T. H. Tatsuishi, Phys. Rev. D 98, 016004 (2018).

[34] J. T. Penedo and S. T. Petcov, Nucl. Phys. B939, 292 (2019).

[35] J. C. Criado and F. Feruglio, SciPost Phys. 5, 042 (2018).

[36] T. Kobayashi, N. Omoto, Y. Shimizu, K. Takagi, M. Tanimoto, and T. H. Tatsuishi, J. High Energy Phys. 11 (2018) 196.

[37] P. P. Novichkov, J. T. Penedo, S. T. Petcov, and A. V. Titov, J. High Energy Phys. 04 (2019) 005; 04 (2019) 174.

[38] H. Okada and M. Tanimoto, Phys. Lett. B 791, 54 (2019).

[39] T. Kobayashi, Y. Shimizu, K. Takagi, M. Tanimoto, T. H. Tatsuishi, and H. Uchida, Phys. Lett. B 794, 114 (2019).
[40] P. P. Novichkov, S. T. Petcov, and M. Tanimoto, Phys. Lett. B 793, 247 (2019).

[41] G. J. Ding, S. F. King, and X. G. Liu, arXiv:1903.12588 [Phys. Rev. D (to be published)].

[42] L. M. Krauss and F. Wilczek, Phys. Rev. Lett. 62, 1221 (1989).

[43] L. E. Ibanez and G. G. Ross, Phys. Lett. B 260, 291 (1991).

[44] T. Banks and M. Dine, Phys. Rev. D 45, 1424 (1992).

[45] T. Araki, T. Kobayashi, J. Kubo, S. Ramos-Sanchez, M. Ratz, and P. K. S. Vaudrevange, Nucl. Phys. B805, 124 (2008).

[46] G. Aldazabal, S. Franco, L. E. Ibanez, R. Rabadan, and A. M. Uranga, J. Math. Phys. (N.Y.) 42, 3103 (2001).

[47] H. Abe, T. Kobayashi, and H. Ohki, J. High Energy Phys. 09 (2008) 043. 